\title{
Numerical Study of Polarization-Dependent Emission Properties of Localized-Surface-Plasmon-Coupled Light Emitting Diodes with $\mathrm{Ag} / \mathrm{SiO}_{2}$ Nanoparticles
}

\author{
Seul-Ki Moon and Jin-Kyu Yang* \\ Department of Optical Engineering, Kongju National University, Cheonan 330-717, Korea
}

(Received July 17, 2014 : revised September 11, 2014 : accepted September 11, 2014)

\begin{abstract}
We study polarization-dependent spontaneous emission (SE) rate and light extraction efficiency (LEE) in localized-surface-plasmon (LSP)-coupled light emitting diodes (LEDs). The closely packed seven $\mathrm{Ag} / \mathrm{SiO}_{2}$ core-shell (CS) nanoparticles (NPs) lie on top of the GaN surface for LSP coupling with a radiated dipole. According to the dipole direction, both the SE rate and the LEE are significantly modified by the LSP effect at the $\mathrm{Ag} / \mathrm{SiO}_{2}$ CS NPs when the size of $\mathrm{Ag}$, the thickness of $\mathrm{SiO}_{2}$, and the position of the dipole source are varied. The enhancement of the SE rate is related to an induced dipole effect at the $\mathrm{Ag}$, and the high LEE is caused by light scattering with an LSP mode at $\mathrm{Ag} / \mathrm{SiO}_{2} \mathrm{CS}$ NPs. We suggest the optimum position of the quantum well (QW) in blue InGaN/GaN LEDs with $\mathrm{Ag} / \mathrm{SiO}_{2}$ CS NPs for practical application.
\end{abstract}

Keywords : Light emitting diodes, Surface plasmons, Spontaneous emission, Light extraction

OCIS codes : (230.3670) Light-emitting diodes; (240.6680) Surface plasmons

\section{INTRODUCTION}

GaN based solid-state light emitting diodes (LEDs) are widely attractive as an alternative energy saving lighting device because of high efficient light emission [1,2]. The external quantum efficiency (EQE) of light emission is given by internal quantum efficiency (IQE) and light extraction efficiency (LEE). For achieving high-efficiency LEDs, both of IQE and LEE should be high enough. The IQE is usually limited by the quality of an active medium such as an InGaN quantum well (QW), and LEE is limited by total internal reflection (TIR) in high index contrast between the semiconductor and surrounding material. Recently, the high IQE of InGaN/GaN blue LEDs was achieved with the help of rapid development of semiconductor growth technology $[3,4]$. The LEE was also dramatically improved by introducing the surface patterning in both the top and bottom surfaces $[5,6]$. However, in green LEDs, the IQE is still very low because of the poor crystal properties such as In dislocation and strong piezo-electric effect [7]. Moreover, in the deep UV regime, there are no transparent electrodes and encapsulation materials, leading to very poor LEE [8].

Since pioneer work in early twenty-first century, surface-
plasmon(SP)-coupled light emitting diodes (LEDs) have been widely studied theoretically and experimentally in order to improve both IQE and LEE, especially in green LEDs [9-13]. The mechanism of IQE enhancement in SP LEDs is the resonant energy transfer from the carrier recombination in QWs to the SP mode at the metal structure, and the outcoupling of the SP mode into the radiation mode. Hence, the efficient QW-SP coupling increases the spontaneous emission (SE) rate, while the nonradiative process is reduced simultaneously. In addition, the metallic nanoparticle (NP) has very large scattering cross section to extract the light outside materials to overcome TIR. For satisfying the industrial demand, it is necessary to achieve not only the high emission efficiency but also no degradation of device performance. However, the problem in Ag NP coated LEDs is an easy oxidation of $\mathrm{Ag}$ which induces significant absorption of the SP mode before transition to the radiation mode. It was reported that $\mathrm{Ag} / \mathrm{SiO}_{2}$ core-shell (CS) coated LEDs had more consistent emission efficiency than Ag NP coated LEDs [14].

Generally, the SE rate of a dipole near a metallic structure strongly depends on the radiation direction [15]. Moreover, the emitted light from the GaN based LEDs is partially polarized according to the growth direction and

\footnotetext{
*Corresponding author: jinkyuyang@kongju.ac.kr

Color versions of one or more of the figures in this paper are available online.
} 
the aluminum content [16-19]. Recently, researchers have paid attention to polarization of light emission in SP-coupled LEDs. Most reports are related to the Ag NP buried in the $\mathrm{p}$-GaN layer, where the enhancement of the radiation power of the dipole near the $\mathrm{Ag} \mathrm{NP}$ is dramatically changed by the direction of the dipole radiation [20-22]. However, the Ag NP embedded structure has several issues for the real LED device because of the irregular Ag NP size caused by the high temperature during the epitaxial process and the degradation of the crystal quality due to the Ag NPs. Moreover, there was no mention about the LEE enhancement because the structure does not include air-GaN boundary structure. One paper reported that the total photoluminescence (PL) decreases significantly by localized SP (LSP) resonant dissipation even though there is large enhancement of photoluminescence (PL) decay rate by the efficient LSP-QW coupling [23]. In this paper, we numerically study polarization dependent enhancement both of the SE rate and the LEE in a closely packed seven $\mathrm{Ag} / \mathrm{SiO}_{2}$ CS NP coated InGaN/GaN LEDs by changing the $\mathrm{Ag}$ size, the thickness of $\mathrm{SiO}_{2}$, and the distance of the QWs from the surface. Finally, we suggested the optimum position of the QW in blue InGaN/GaN LEDs with $\mathrm{Ag} / \mathrm{SiO}_{2}$ CS NPs for practical application.

\section{NUMERICAL METHODS}

Recently, the dipole radiation property near Ag NP was studied by semi-classical analysis [20, 21]. According to the reports, the SP mode could be analyzed by the theoretical model when the Ag NPs are in homogeneous media. However, it is hard to solve the optical property of $\mathrm{Ag} / \mathrm{SiO}_{2}$ CS NP coated InGaN/GaN LEDs by the semi-classical approach because of breaking the azimuthal symmetry of the structure. In this paper, we analyze light emission properties by a full three-dimensional (3-D) finite-difference time-domain (FDTD) method [24, 25].

The schematic cross section of the LED structure being

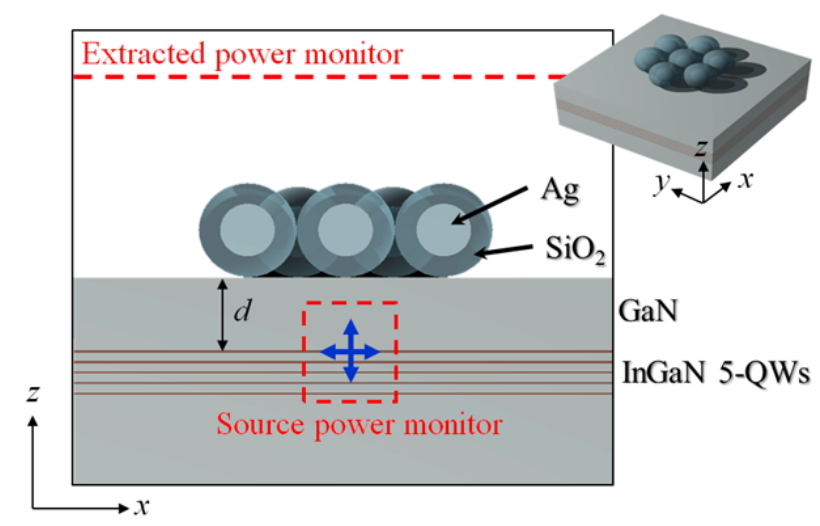

FIG. 1. Schematic cross section of $\mathrm{Ag} / \mathrm{SiO}_{2} \mathrm{CS} \mathrm{NPs}$ on InGaN/GaN LEDs. considered is shown in Fig. 1. The closely packed seven identical $\mathrm{Ag} / \mathrm{SiO}_{2} \mathrm{CS}$ NPs lie on the p-GaN surface. The five InGaN QWs are positioned below the 70-nm-thick p-GaN layer. Each QW has the same thickness with $2 \mathrm{~nm}$ and the barrier between QWs is $8 \mathrm{~nm}$. The refractive indices of $\mathrm{InGaN}$ and $\mathrm{GaN}$ are 2.7 and 2.46, respectively. Here, we use the perfectly matched layer (PML) absorbing boundary to realize the infinite-size space. The spatial nonuniform-grid FDTD method is used for saving the computational memory. Note that the minimum grid size is fixed as $1 \mathrm{~nm}$, which is an adequate size to resolve the strongly localized field distribution in consideration of limited computational power. The dipole polarized parallel to the surface (TE-polarized dipole) or normal to the surface (TMpolarized dipole) is launched at the center of the 2-nm-thick InGaN QW. The lateral position of the dipole source is fixed at the center of the central $\mathrm{Ag} / \mathrm{SiO}_{2} \mathrm{CS} \mathrm{NP}$. The total radiation power is obtained by integrating the power flux through the monitor box surrounding the dipole source. And the extracted power is obtained by integrating the power flux through the monitor plane at $135 \mathrm{~nm}$ above the $\mathrm{p}$-GaN surface, which corresponds around a 74-degree solid angle. The enhancement of the SE rate $\left(\gamma^{\mathrm{rad}} / \gamma_{0}^{\mathrm{rad}}\right)$ and the enhancement of the LEE $\left(\eta^{\text {ext }} / \eta_{0}^{\text {ext }}\right)$ was achieved by normalizing the total radiation power and the extracted power with those at the LED structure without $\mathrm{Ag} / \mathrm{SiO}_{2}$ CS NPs. In all simulations, we fixed the number of $\mathrm{Ag} / \mathrm{SiO}_{2}$ CS NPs as seven, which is the optimum condition for maximizing the extracted power [26].

\section{RESULTS AND DISCUSSION}

\subsection{Dependence On The Ag Size}

The LSP resonance is strongly affected by the size, the shape and the environmental materials of the metal NP [27]. Therefore, in order to improve the emission efficiency of the LEDs, the LSP resonance should be spectrally close to the emission wavelength. First, we investigated the Ag size dependence of the SE rate and the LEE with different dipole polarizations. Here, the thickness of $\mathrm{SiO}_{2}$ is fixed at $20 \mathrm{~nm}$, and the source distance from the surface, $d$ is fixed at $71 \mathrm{~nm}$, the center of the first QW.

Figure 2(a) shows $\gamma^{\mathrm{rad}} / \gamma_{0}^{\text {rad }}$ as a function of wavelength by changing the radius of Ag. The upper graph corresponds to the TM-polarized dipole and the lower corresponds to the TE-polarized dipole. Both the TM-polarized dipole and the TE-polarized dipole have a dip near $425 \mathrm{~nm}$ in spectra of the SE rate. As the radius of $\mathrm{Ag}$ increases, the dip becomes deeper and wider with a small red-shift. This is related to the nonradiative decay rate by the $\mathrm{Ag} / \mathrm{SiO}_{2} \mathrm{CS}$ NPs. On the other hand, the peak is only found in the TE-polarized dipole at the longer wavelength of the dip. The origin of the enhancement of the SE rate is near-field energy coupling between the dipole emitter and the LSP mode [9-11]. We will discuss this in the next paragraph 


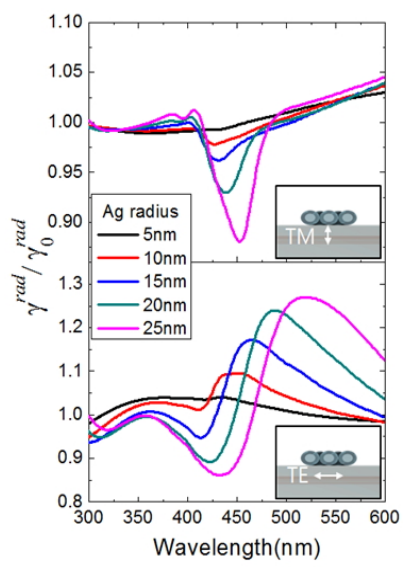

(a)

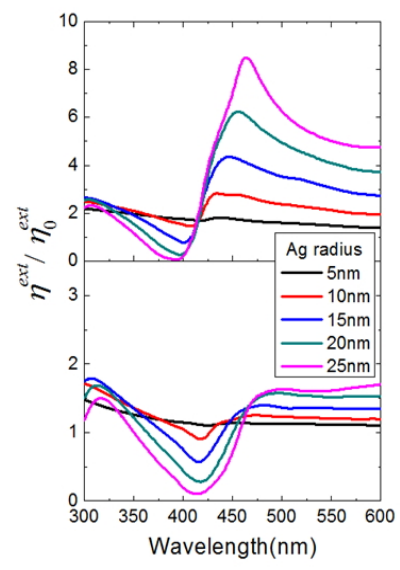

(b)
FIG. 2. Dependence of (a) $\gamma^{\text {rad }} / \gamma_{0}^{\text {rad }}$ and (b) $\eta^{\text {ext }} / \eta_{0}^{\text {ext }}$ on the radius of $\mathrm{Ag}$ cores at $\mathrm{Ag} / \mathrm{SiO}_{2} \mathrm{CS}$ coated InGaN/GaN LED structure. The upper graphs are for the TM-polarized dipole and the lower graphs are for the TE-polarized dipole. All spectral results are normalized by the results at the LED structure without the $\mathrm{Ag} / \mathrm{SiO}_{2} \mathrm{CS}$ NPs.

and in Sec. 3.3 in details. In Fig. 2(b), $\eta^{\text {ext }} / \eta_{0}^{\text {ext }}$ at different sizes of the $\mathrm{Ag}$ are plotted as a function of the wavelength. As the radius of $\mathrm{Ag}$ becomes large, it is overall enhanced in the long wavelength region at both polarizations. As the size of the $\mathrm{Ag}$ increases, the modal volume of the LSP resonance becomes large, so the coupling efficiency between the emitted light and the LSP mode increases. The coupled LSP mode is converted back into the radiative light. The significant reduction of the LEE is observed at a short wavelength, $\sim 425 \mathrm{~nm}$ in both dipoles as the radius of $\mathrm{Ag}$ increases. This is related to the absorption of the coupled LSP mode by ohmic loss. The peak of $\eta^{\text {ext }} / \eta_{0}^{\text {ext }}$ in the TM-polarized dipole is more pronounced with the red-shift. This is related to the inherent radiation direction. Because the TM-polarized dipole is radiated along the in-plane direction, most of the radiated light could not escape from the GaN substrate because of TIR. Therefore, even if a small part of the light coupled to the LSP mode, the enhancement of LEE could be large. The line-shape of $\eta^{\text {ext }} / \eta_{0}^{\text {ext }}$ looks like the Fano-resonance, which is caused by the temporal interference between the direct radiation from the dipole and the re-radiated light from the coupled LSP mode $[21,28]$.

For the further understanding of the SE rate change, we considered a simple model: a dipole emitter near a metal sphere in a homogeneous medium. This problem has an exact analytical solution based on Mie scattering theory [28-30]. The presence of the metallic NP near a dipole emitter introduces an additional nonradiative decay channel which depends only on the material property of the metal. Therefore, as the size of $\mathrm{Ag}$ increases, the nonradiative decay rate is enhanced, while there is no significant polarization dependence and no wavelength shift [31]. This is matched with the tendency of the dip in spectra of $\gamma^{\mathrm{rad}} / \gamma_{0}^{\mathrm{rad}}$ in Fig. 2(a). In contrast, when the dipole emitter is close to the metallic structure, the radiative decay rate can be also dramatically changed by the coupling effect between the dipole and the induced dipole (the so-called surface plasmon mode) according to the orientation of the dipole moment $[29,31]$.

The strength of the resonant coupling is determined by how the dipole emitter is close to the LSP mode, spatially and spectrally. We also calculated the absorption and resonance behaviors at $\mathrm{Ag} / \mathrm{SiO}_{2} \mathrm{CS}$ NPs in both the TM-polarized dipole and the TE-polarized dipole. Figure 3(a) shows the absorption spectra when the radius of $\mathrm{Ag}$ is $15 \mathrm{~nm}$. The absorption spectrum is calculated by subtracting the Poynting flux from the total radiation power of the dipole source. Here, the boundary of the calculation domain with the exception of the PML layer is used as the integral surface of the Poynting flux, which should be far from a dipole source and the LSP mode at the $\mathrm{Ag} / \mathrm{SiO}_{2}$ CS NPs. Both the TM-polarized dipole and the TE-polarized dipole have a similar absorption spectrum. The difference of the absorption intensity between the TM-polarized dipole and the TE-polarized dipole is caused by different radiation direction. Figure 3 (b) shows the LSP resonance spectra at $\mathrm{Ag} / \mathrm{SiO}_{2} \mathrm{CS}$ NPs when the dipole source is launched at 71 $\mathrm{nm}$ below the $\mathrm{GaN}$ surface. Note that the resonance spectrum is obtained by the discrete Fourier transform of the time-varying electric field at the maximum intensity positions after the dipole source radiation which are marked as the red dot (the left edge of the center $\mathrm{Ag}$ ) for the TM-polarized dipole and the black dot (the top of the center $\mathrm{Ag}$ ) for the TE-polarized dipole, as shown in the inset of Fig. 3(b). The resonance spectra look very similar to the absorption spectra. This implies that the absorption of the radiative light is caused by the ohmic loss after coupling with the LSP modes in $\mathrm{Ag} / \mathrm{SiO}_{2}$ CS NPs. Two LSP resonance peaks are found at $428 \mathrm{~nm}$, and $503 \mathrm{~nm}$ regardless of the dipole direction. Figure 3(c) show the cut-views of the electric field distribution of the LSP mode at $428 \mathrm{~nm}$ in the TM-polarized dipole. According to the xz cut-view, the electric field has a symmetric distribution along the z-axis. In addition, from the xy cut-view, the electric field near the center of $\mathrm{Ag} / \mathrm{SiO}_{2} \mathrm{CS} \mathrm{NP}$ is oscillated in the radial direction. In case of the LSP resonance in the TE-polarized dipole, the direction of the electric field near the left and right $\mathrm{Ag} / \mathrm{SiO}_{2}$ is along the x-direction shown in the left of Fig. 3 (d). Moreover, the electric field intensity near three $\mathrm{Ag} / \mathrm{SiO}_{2} \mathrm{CS}$ NPs on the $\mathrm{x}$-axis is stronger than the intensity near the others, as shown in the xy cut-view. From these facts, the LSP resonance in the TE-polarized dipole is the coupled $\mathrm{x}$-dipole modes at $\mathrm{Ag} / \mathrm{SiO}_{2} \mathrm{CS} \mathrm{NPs}$ on the $\mathrm{x}$-axis. In the $\mathrm{TM}$ resonance, the intensity at the $\mathrm{GaN}$ layer underneath the center $\mathrm{Ag} / \mathrm{SiO}_{2} \mathrm{NP}$ is stronger than that in the TE resonance. Hence, the resonant energy transfer between the QW and the LSP in the TM-polarized dipole is more 
efficient than in the TE-polarized dipole. We will discuss

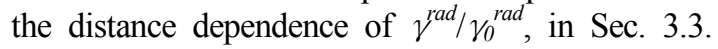

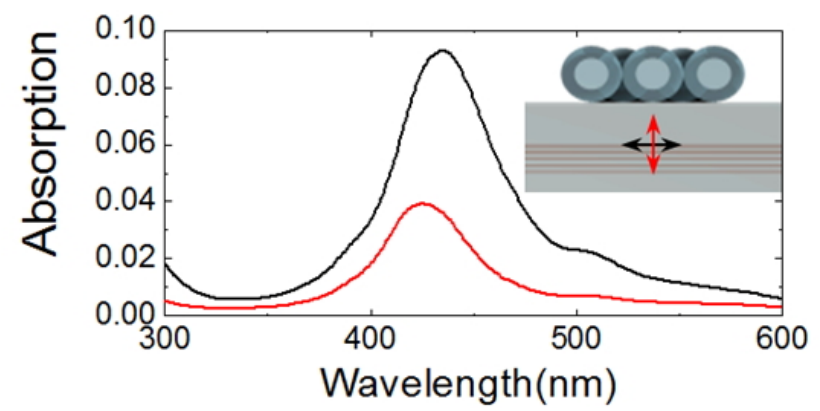

(a)

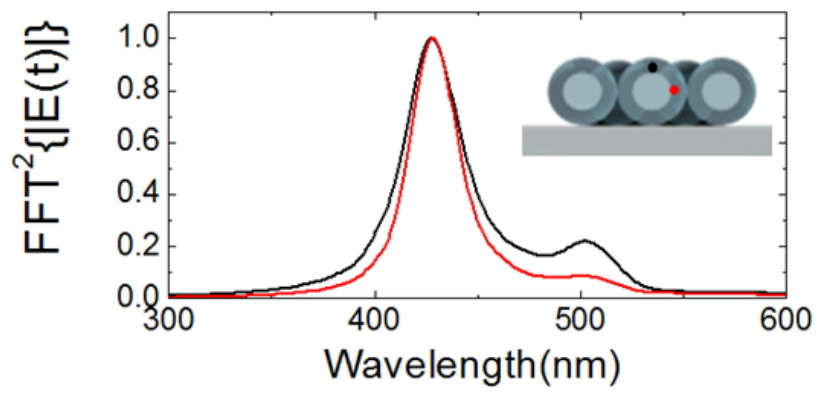

(b)
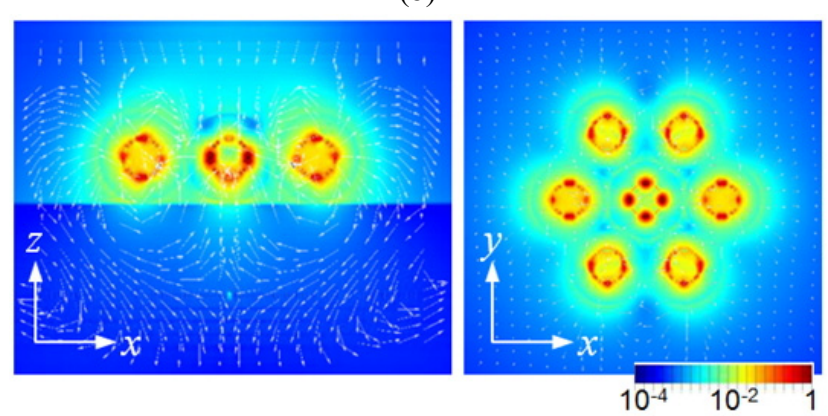

(c)
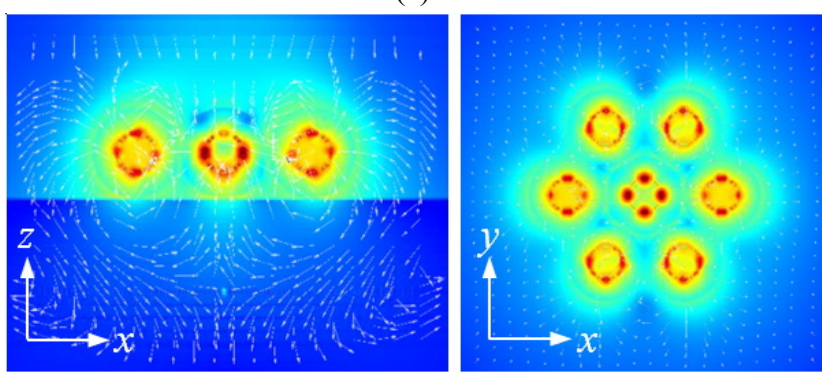

(d)

FIG. 3. LSP absorption and resonance behavior at seven $\mathrm{Ag} / \mathrm{SiO}_{2} \mathrm{CS}$ NPs on the GaN surface. (a) Absorption spectra (b) Resonance spectra (c) Electric field distribution at the resonance, $\lambda=428 \mathrm{~nm}$ in the TM-polarized dipole excitation. The left is the xz cut-view of electric field. The right is the xy cut-view of the electric field. (d) Electric field distribution at the resonance, $\lambda=428 \mathrm{~nm}$ in the TE-polarized dipole excitation. The arrows in (c) and (d) indicate the direction of the electric field.

\subsection{Dependence On The $\mathrm{SiO}_{2}$-shell Thickness}

Usually, the Ag NPs are easily oxidized at the surface as time goes on. Therefore, the LSP absorption in the Ag $\mathrm{NP}$ is dramatically enhanced by the ohmic loss in the Ag surface [14]. In order to avoid the absorption in the $\mathrm{Ag}$ surface, the $\mathrm{Ag} / \mathrm{SiO}_{2} \mathrm{CS}$ structure was suggested for the efficient and reliable SP LED performance. However, if the $\mathrm{SiO}_{2}$ shell is too thick, $\gamma^{\mathrm{rad}} / \gamma_{0}^{\text {rad }}$ and $\eta^{\text {ext }} / \eta_{0}^{\text {ext }}$ become low because of the weak coupling between the dipole emitter and the LSP mode. In addition, the resonance wavelength of the LSP mode is changed by effective index change due to the different thicknesses of the $\mathrm{SiO}_{2}$ shell [27]. In this section, we systematically investigated $\gamma^{r a d} / \gamma_{0}^{r a d}$ and $\eta$ ${ }^{e x t} / \eta_{0}{ }^{e x t}$ with the TM-polarized and the TE-polarized dipole sources as the thickness of $\mathrm{SiO}_{2}$ is varied from $5 \mathrm{~nm}$ to 20 $\mathrm{nm}$. In this calculation, we fixed the $\mathrm{Ag}$ radius at $15 \mathrm{~nm}$ and the source distance at $71 \mathrm{~nm}$ from the surface.

Figure 4(a) shows the spectra of $\gamma^{\mathrm{rad}} / \gamma_{0}^{\mathrm{rad}}$ with various thicknesses of $\mathrm{SiO}_{2}$. The upper graph corresponds to the TM-polarized dipole radiation. As the thickness increases, a spectral dip is red-shifted, which is caused by increasing effective index [27]. And also, the distance from the dipole source to the $\mathrm{Ag}$ increases, so the nonradiative decay rate by the $\mathrm{Ag}$ is reduced and the resonant energy transfer between the dipole emitter and the LSP mode becomes weak. Hence, as the $\mathrm{SiO}_{2}$ shell thickens, the $\gamma^{\mathrm{rad} /}$ $\gamma_{0}^{r a d}$ curve becomes flatter. Similarly, for the TE-polarized dipole source (shown in the lower graph of Fig. 4(a)), as the thickness of $\mathrm{SiO}_{2}$ increases, the peak is red-shifted and decreases. We also investigated the dependence of the $\mathrm{LEE}$ on $\mathrm{SiO}_{2}$ shell thickness with different polarizations of the dipole source. The upper graph in Fig. 4(b) corresponds to $\eta^{\text {ext }} / \eta_{0}{ }^{\text {ext }}$ in the TM-polarized dipole source. As the $\mathrm{SiO}_{2}$ shell becomes thick, the enhancement of the LEE decreases due to the weak scattering at the LSP mode, and the peak wavelength is red-shifted owing to increasing effective

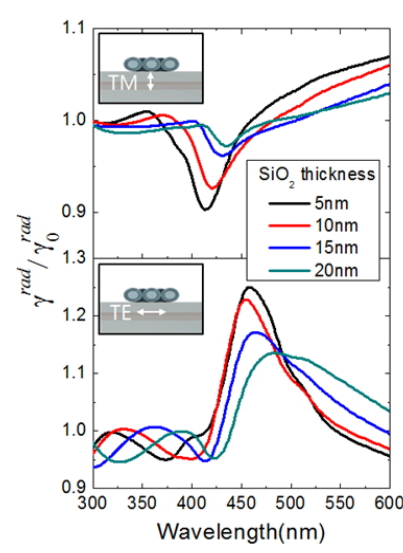

(a)

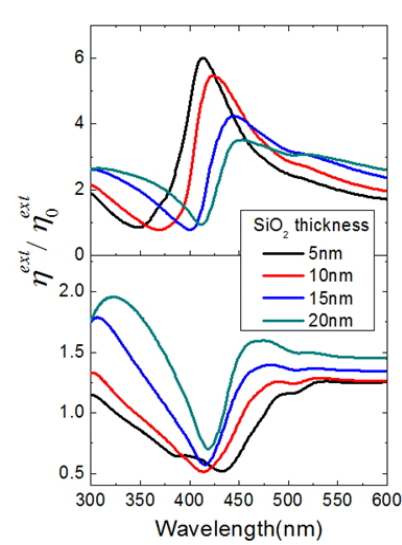

(b)
FIG. 4. Dependence of (a) $\gamma^{r a d} / \gamma_{0}{ }^{r a d}$ and (b) $\eta^{\text {ext }} / \eta_{0}{ }^{e x t}$ on the $\mathrm{SiO}_{2}$-shell thickness at $\mathrm{Ag} / \mathrm{SiO}_{2} \mathrm{CS}$ coated InGaN/GaN LED structure. The upper graphs are for the TM-polarized dipole and the lower graphs are for the TE-polarized dipole. 
index. In contrast, in the TE-polarized dipole, as the thicker $\mathrm{SiO}_{2}$, the more light is extracted from the $\mathrm{GaN}$ as shown in the lower graph of Fig. 4(b). The TE-polarized dipole has a large vertical radiation component, so $\mathrm{Ag} / \mathrm{SiO}_{2} \mathrm{CS}$ NPs act as strong scatterers. Therefore, in a 5-nm thickness, the LEE decreases significantly. As the $\mathrm{SiO}_{2}$ shell becomes thick, the separation distance between the Ag cores increases, hence, the scattering of the emitted light is reduced. Moreover, the thick $\mathrm{SiO}_{2}$ shell helps to enhance the light extraction because of the increase in the critical angle by inserting a middle-index layer between high-index material and low-index material.

\subsection{Dependence On The Quantum-well Position}

The most important parameter for the efficient QW-SP coupling is the distance from the metallic NPs to the emitter $[10,30-31]$. If the dipole emitter is close to the NPs, the quantum efficiency increases because of the stronger QW-SP coupling and light scattering. However, if the dipole emitter is very close, about a few nm, the quantum yield decreases rapidly, hence the quantum efficiency decreases by the quenching effect $[28,31]$. In this section, we systematically studied the dependence of the quantum efficiency on the distance between QWs and $\mathrm{Ag} / \mathrm{SiO}_{2} \mathrm{CS}$ NPs according to the dipole direction. Here, we fixed the radius of $\mathrm{Ag}$ and the thickness of $\mathrm{SiO}_{2}$ as $15 \mathrm{~nm}$ and 20 $\mathrm{nm}$, respectively.

Figure 5(a) shows the dependence of $\gamma^{\mathrm{rad}} / \gamma_{0}^{\mathrm{rad}}$ on the distance, $d$, from the GaN surface to the dipole emitter. In case of the TM-polarized dipole shown in the upper graph at Fig. 5(a), $\gamma^{\mathrm{rad}} / \gamma_{0}^{\text {rad }}$ is dramatically enhanced at all wavelengths as $d$ decreases, especially, $\gamma^{r a d} / \gamma_{0}^{r a d} \sim 7$ near the LSP resonance for $d=11 \mathrm{~nm}$. This high enhancement is owing to the strong coupling between the dipole and the LSP mode. However, as $d$ increases, $\gamma^{\text {rad }} / \gamma_{0}^{\text {rad }}$ goes to unity quickly. On the other hand, the SE rate in the TE-polarized dipole decreases at all wavelengths at $d=11$ $\mathrm{nm}$, as shown in the lower graph at Fig. 5(a). As $d$ increases, $\gamma^{r a d} / \gamma_{0}^{r a d}$ goes to unity with some fluctuation in wavelength.

This phenomenon could be understood by using the electrostatic image theory [28, 33]. According to the method of image charges, the image dipole moment, $\boldsymbol{p}_{\mathrm{i}}$ $\boldsymbol{p}_{\mathrm{o}} \times(r / d)^{3}$, where $\boldsymbol{p}_{\mathrm{o}}$ is the source dipole moment, $\mathrm{r}$ is the radius of the metal sphere, and $d$ is the distance from the center of the metal sphere to the position of a dipole source. In the TM-polarized dipole, the direction of the dipole oscillation is perpendicular to the metallic surface, therefore, the induced dipole has the same direction as that of the source. Hence, as $d$ is very small, the radiative decay rate increases dramatically. However, as $d$ increases, the induced dipole becomes weak quickly, so the SE rate approaches to that in the bulk GaN. This analysis agrees well with the intensity distribution of LSP resonance shown in Fig. 3(c). In the case of the TE-polarized dipole, the direction of the dipole oscillation is parallel to the metallic surface, and the induced dipole has the opposite direction to the source dipole. Therefore, if the source dipole is close to the metal sphere, two dipoles are cancelled out, and its radiation efficiency is reduced. However, as $d$ increases, the SE rate is changed by the interference effect between the source dipole and the induced dipole.

Figure 5(b) shows the dependence of $\eta^{\text {ext }} / \eta_{0}^{\text {ext }}$ on the distance as a function of wavelength for the TM-polarized dipole (the upper graph) and the TE-polarized light (the lower graph). For both polarizations, as $d$ increases, $\eta^{\text {ext }} / \eta_{0}^{\text {ext }}$ increases near the LSP resonance $(\sim 25 \mathrm{~nm})$, which is related with reduction of the absorption by decreasing the coupling efficiency between the emitted light and the LSP mode. As we mentioned above, the Fano-line shape near the LSP resonance is owing to the temporal interference between the direct radiation from the dipole and scattered light to the LSP mode [28]. At $d=11 \mathrm{~nm}$, the LEE in the TM-polarized dipole is significantly suppressed. However, as $d$ increases, $\eta^{\text {ext }} / \eta_{0}^{\text {ext }}$ is dramatically enhanced and then decreases. This is originated from interplay between the re-radiation of the coupled LSP mode and the absorption by ohmic loss. As the dipole is far from the metal NPs, the light scattering to the LSP mode decreases, and the ohmic loss is reduced. Therefore, there is an optimum distance to maximize the LEE. In the case of the TE-polarized dipole, we observed two-fold enhancement of the LEE near $475 \mathrm{~nm}$ at $d=11 \mathrm{~nm}$. This is due to the strong coupling of the emitted light with the LSP mode. As $d$ increases, $\eta^{\text {ext }} / \eta_{0}^{\text {ext }}$ decreases, and then increases at $d>71 \mathrm{~nm}$.

According to these results, as $d$ increases, the trend of the SE rate change is opposite to that of the LEE variation in both TM- and TE-polarized dipoles. For the practical application of the blue LED $(\lambda=450 \mathrm{~nm} \pm 15 \mathrm{~nm})$, we calculated the enhancement of the total extracted power as a function of $d$ as shown in Fig. 6. First, in the TM-polarized dipole, the total extracted power dramatically

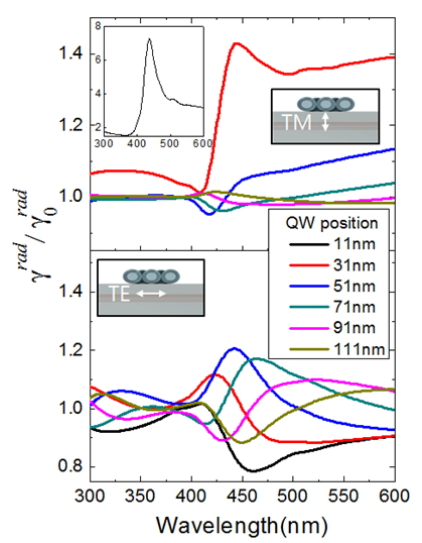

(a)

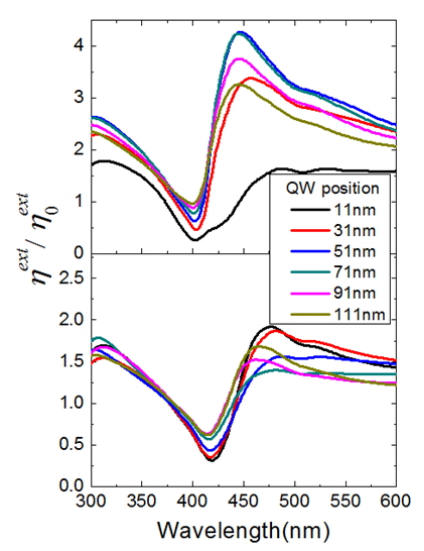

(b)
FIG. 5. Dependence of (a) $\gamma^{r a d} / \gamma_{0}^{r a d}$ and (b) $\eta^{\text {ext }} / \eta_{0}^{e x t}$ on QW position at $\mathrm{Ag} / \mathrm{SiO}_{2}$ CS NP coated InGaN/GaN LED structure. The upper graphs are for the TM-polarized dipole and the lower graphs are for the TE-polarized dipole. 


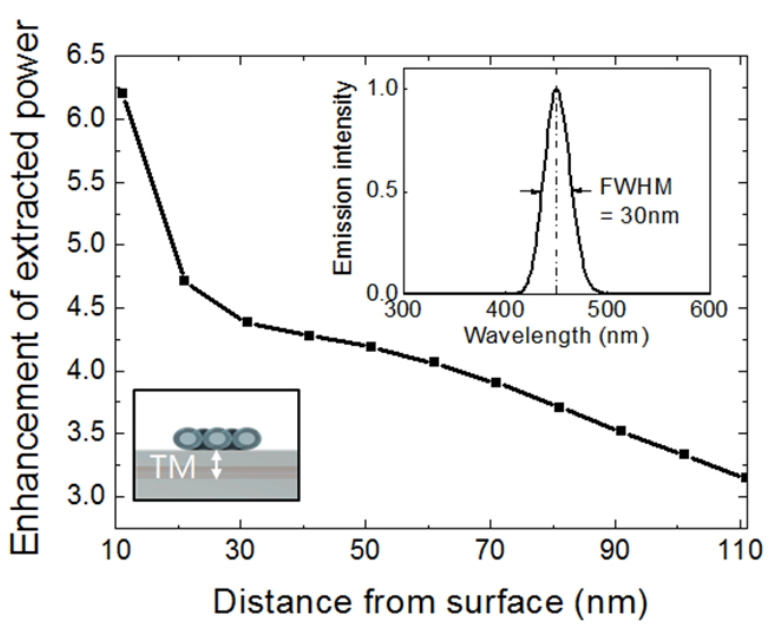

(a)

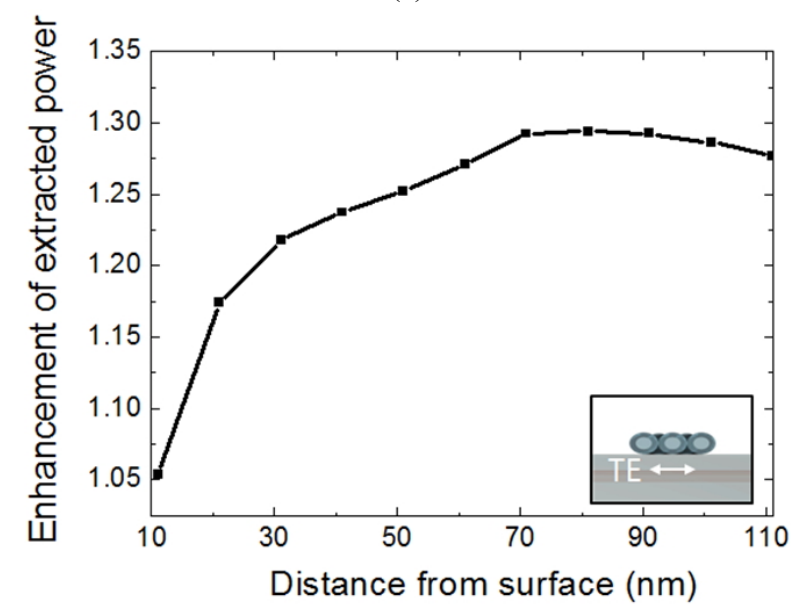

(b)

FIG. 6. The enhancement of extracted power as a function of the QW distance from the top surface of $\mathrm{Ag} / \mathrm{SiO}_{2} \mathrm{CS}$ coated InGaN/GaN blue LEDs at (a) TM-polarized dipole and (b) TE-polarized dipole. The inset graph at (a) indicates the spectrum of a reference LED.

increases at $d=11 \mathrm{~nm}$ because of the great enhancement of the SE rate by strong coupling between the dipole and the LSP mode. As $d$ increases, $\gamma^{\text {rad }} / \gamma_{0}^{\text {rad }}$ goes to unity quickly. On the contrary, $\eta^{\text {ext }} / \eta_{0}^{\text {ext }}$ increases until $d=71$ $\mathrm{nm}$, and then decreases with further increasing $d$. Hence, the enhancement of the total extracted power gradually decreases. On the other hand, in the TE-polarized dipole, the total extracted power is maximized about 1.3 times higher than that in the blue LED without $\mathrm{Ag} / \mathrm{SiO}_{2} \mathrm{CS}$ NPs at $\sim 80 \mathrm{~nm}$ of the QW from the surface. The overall enhancement of the total extracted power in the TM-polarized is much higher than in the TE-polarized dipole. However, because it is very hard to extract the TM-polarized light due to its inherent radiation direction, the absolute extracted power is still much smaller than that in the TE-polarized light.

\section{CONCLUSION}

We numerically investigated polarization-dependent SE rate and LEE in LSP-coupled LEDs. The closely packed seven $\mathrm{Ag} / \mathrm{SiO}_{2}$ CS NPs lie on top of the GaN surface for LSP coupling with a radiated dipole. According to the dipole direction, both the SE rate and the LEE are significantly modified by LSP effect at $\mathrm{Ag} / \mathrm{SiO}_{2}$ CS NPs when the size of $\mathrm{Ag}$, the thickness of $\mathrm{SiO}_{2}$, and the position of the dipole source are varied. In the dipole oscillating parallel to the surface, the SE rate is modified by strong near-field energy coupling and the interference effect between a real dipole and an induced dipole at the $\mathrm{Ag}$, and the LEE is changed due to light scattering and ohmic loss at $\mathrm{Ag} / \mathrm{SiO}_{2}$ CS NPs. In the dipole oscillating normal to the surface, the SE rate is dramatically enhanced by extremely strong near-field coupling only when the dipole is near the Ag. And the LEE is significantly enhanced because the light mostly guided in the substrate is extracted by scattering with LSP modes. Finally we suggested the optimum QW position for maximizing extracted power in blue InGaN/GaN LEDs at each dipole direction. In TM polarization, the extracted power keeps increasing as the QW is close to the $\mathrm{Ag} / \mathrm{SiO}_{2} \mathrm{CS} \mathrm{NPs}$. However, in TE polarization, the extracted power is maximized about 1.3 times higher than that in the blue LED without $\mathrm{Ag} / \mathrm{SiO}_{2} \mathrm{CS}$ NPs when the QW is near 80 $\mathrm{nm}$ from the surface. We believe that these results help to optimize the nitride-based LSP-LED structures with different growth directions.

\section{ACKNOWLEDGMENT}

The authors thank Prof. In-Hwan Lee for stimulating discussions. This work was supported by the research grant of the Kongju National University in 2013.

\section{REFERENCES}

1. E. F. Schubert, Light-Emitting Diodes (Cambridge University Press, Cambridge, 2006).

2. M. H. Crawford, "LEDs for solid-state lighting: Performance challenges and recent advances," IEEE J. Select. Topics Quantum Electron. 15, 1028-1040 (2009).

3. D. F. Feezell, J. S. Speck, S. P. DenBaars, and S. Nakamura, "Semipolar (20 $2 \overline{1}$ ) InGaN/GaN light-emitting diodes for high-efficiency solid-state lighting," J. Disp. Technol. 9, 190-198 (2013).

4. M. J. Cich, R. I. Aldaz, A. Chakraborty, A. David, M. J. Grundmann, A. Tyagi, M. Zhang, F. M. Steranka, and M. R. Krames, "Bulk GaN based violet light-emitting diodes with high efficiency at very high current density," Appl. Phys. Lett. 101, 223509 (2012). 
5. J. J. Wierer, A. David, and M. M. Megens, "III-nitride photonic-crystal light-emitting diodes with high extraction efficiency," Nat. Photon. 3, 163-169 (2009).

6. T.-X. Lee, K.-F. Gao, W.-T. Chien, and C.-C. Sun, "Light extraction analysis of GaN-based light-emitting diodes with surface texture and/or patterned substrate," Opt. Express 15, 6670-6676 (2007).

7. U. T. Schwarz, H. Braun, K. Kojima, Y. Kawakami, S. Nagahama, and T. Mukai, "Interplay of built-in potential and piezoelectric field on carrier recombination in green light emitting InGaN quantum wells,” Appl. Phys. Lett. 91, 123503 (2007).

8. A. Khan, K. Balakrishnan, and T. Katona, "Ultraviolet light-emitting diodes based on group three nitrides," Nat. Photon. 2, 77-84 (2008).

9. J. Vuckovic, M. Loncar, and A. Scherer, "Surface plasmon enhanced light-emitting diode," IEEE J. Quantum Electron. 36, 1131-1144 (2000).

10. K. Okamoto, I. Niki, A. Shvartser, Y. Narukawa, T. Mukai, and A. Scherer, "Surface-plasmon-enhanced light emitters based on InGaN quantum wells," Nat. Mater. 3, 601-605 (2004).

11. K. Okamoto, I. Niki, A. Scherer, Y. Narukawa, T. Mukai, and Y. Kawakami, "Surface plasmon enhanced spontaneous emission rate of $\mathrm{InGaN} / \mathrm{GaN}$ quantum wells probed by time-resolved photoluminescence spectroscopy," Appl. Phys. Lett. 87, 071102 (2005).

12. M. K. Kwon, J. Y. Kim, B. H. Kim, I. K. Park, C. Y. Cho, C. C. Byeon, and S. J. Park, "Surface-plasmon-enhanced light-emitting diodes," Adv. Mater. 20, 1253-1257 (2008).

13. C.-H. Lu, C.-C. Lan, Y.-L. Lai, Y.-L. Li, and C.-P. Liu, "Enhancement of green emission from InGaN/GaN multiple quantum wells via coupling to surface plasmons in a twodimensional silver array," Adv. Funct. Mater. 21, 4719-4723 (2011).

14. L.-W. Jang, D.-W. Jeon, M. Kim, J.-W. Jeon, A. Y. Polyakov, J.-W. Ju, S.-J. Lee, J.-H. Baek, J.-K. Yang, I.-H. Lee, "Investigation of optical and structural stability of localized surface plasmon mediated light-emitting diodes by $\mathrm{Ag}$ and $\mathrm{Ag} / \mathrm{SiO}_{2}$ nanoparticles," Adv. Func. Mater. 22, 2728-2734 (2012).

15. Y. Xu, J. Vuckovic, R. Lee, O. Painter, A. Scherer, and A. Yariv, "Finite-difference time-domain calculation of spontaneous emission lifetime in a microcavity," J. Opt. Soc. Am. B 16, 465-474 (1999).

16. N. F. Gardner, J. C. Kim, J. J. Wierer, Y. C. Shen, and M. R. Krames, "Polarization anisotropy in the electroluminescence of m-plane InGaN-GaN multiple-quantum-well light-emitting diodes," Appl. Phys. Lett. 86, 111101 (2005).

17. H. Masui, T. J. Baker, M. Iza, H. Zhong, S. Nakamura, and S. P. DenBaars, "Light-polarization characteristics of electroluminescence from $\mathrm{InGaN} / \mathrm{GaN}$ light-emitting diodes prepared on (11 $\overline{2} 2$ )-plane GaN," J. Appl. Phys. 100, 113109 (2006).

18. M. F. Schubert, S. Chhajed, J. K. Kim, and E. F. Schubert, "Polarization of light emission by $460 \mathrm{~nm} \mathrm{GaInN/GaN}$ light-emitting diodes grown on (0001) oriented sapphire substrates," Appl. Phys. Lett. 91, 051117 (2007).
19. T. Kolbe, A. Knauer, C. Chua, Z. Yang, S. Einfeldt, P. Vogt, N. M. Johnson, M. Weyers, and M. Kneissl, "Optical polarization characteristics of ultraviolet $(\mathrm{In})(\mathrm{Al}) \mathrm{GaN}$ multiple quantum well light emitting diodes," Appl. Phys. Lett. 97, 171105 (2010).

20. Y. Kuo, S. Y. Ting, C. H. Liao, J. J. Huang, C. Y. Chen, C. Hsieh, Y. C. Lu, C. Y. Chen, K. C. Shen, C. F. Lu, D. M. Yeh, J. Y. Wang, W. H. Chuang, Y. W. Kiang, and C. C. Yang, "Surface plasmon coupling with radiating dipole for enhancing the emission efficiency of a light-emitting diode," Opt. Express 19, A914-A929 (2011).

21. Y. Kuo, W. Y. Chang, H. S. Chen, Y. W. Kiang, and C. C. Yang, "Surface plasmon coupling with a radiating dipole near an Ag nanoparticle embedded in GaN," Appl. Phys. Lett. 102, 161103 (2013).

22. Y. Kuo, W. Y. Chang, H. S. Chen, Y. R. Wu, C. C. Yang, and Y. W. Kiang, "Surface-plasmon-coupled emission enhancement of a quantum well with a metal nanoparticle embedded in a light-emitting diode," J. Opt. Soc. Am. B 30, 2599-2606 (2013).

23. S. Jiang, Z. Hu, Z. Chen, X. Fu, X. Jiang, Q. Jiao, T. Yu, and G. Zhang, "Resonant absorption and scattering suppression of localized surface plasmons in Ag particles on green LED," Opt. Express 21, 12100-12110 (2013).

24. A. Taflove and S. Hagness, Computational Electrodynamics: The Finite-Difference Time-Domain Method (Artech House Publishers, Boston, USA, 2000).

25. http://www.lumerical.com/

26. S.-K. Moon and J.-K. Yang, "Effect of number of $\mathrm{Ag} / \mathrm{SiO}_{2}$ core-shells on quantum efficiency of $\mathrm{InGaN} / \mathrm{GaN}$ light emitting diodes," in preparation (2014).

27. K. L. Kelly, E. Coronado, L. L. Zhao, and G. C. Schatz, "The optical properties of metal nanoparticles: The influence of size, shape, and dielectric environment," J. Phys. Chem. B 107, 668-677 (2003).

28. H. Mertens, A. F. Koenderink, and A. Polman, "Plasmonenhanced luminescence near noble-metal nanospheres: Comparison of exact theory and an improved Gersten and Nitzan model," Phys. Rev. B 76, 115-123 (2007).

29. Y. S. Kim, P. T. Leung, and T. F. George, "Classical decay rates for molecules in the presence of a spherical surface: a complete treatment," Surf. Sci. 195, 1-14 (1988).

30. G. Colas des Francs, A. Bouhelier, E. Finot, J. C. Weeber, A. Dereux, C. Girard, and E. Dujardin, "Fluorescence relaxation in the near-field of a mesoscopic metallic particle: Distance dependence and role of plasmon modes," Opt. Express 16, 17654-17666 (2008).

31. Bharadwaj and L. Novotny, "Spectral dependence of single molecule fluorescence enhancement," Opt. Express 15, 14266-14274 (2007).

32. I. Abram, I. Rovert, and R. Kuszelwicz, "Spontaneous emission control in semiconductor microcavities with metallic or Bragg mirrors," IEEE J. Quantum Electron. 34, 71-76 (1998).

33. Z. Zhang, D. Lim, and R. E. Diaz, "Image theory for plasmon-modified luminescence near nanospheres," Proc. SPIE 8595, 859508 (2013). 